\title{
Evaluation of the Participant-Support Method for Information Acquisition in the "Multiplex Risk Communicator"
}

\author{
Hiroshi Yajima*, Tomohiro Watanabe, and Ryoichi Sasaki* \\ School of Engineering, Tokyo Denki University \\ 2-2 Kandanishiki-cho Chiyoda-ku Tokyo \\ * RISTEX of the Japan Science and Technology Agency \\ yajima@im.dendai.ac.jp, sasaki@im.dendai.ac.jp
}

\begin{abstract}
There has been a diversification of the social risks to informationbased society leading to complex social issues, and risk communication is necessary in order to solve the complicated social problems that arise concerning stakeholders with various levels of knowledge and differing standards regarding risks. In this paper, we introduce a portal system that assists participants to achieve an optimal combination of countermeasures. The characteristics of the proposed method are: first, to classify the stage when participants acquire information; second, to enable smooth transitions during the information acquisition stage; and third, to support information acquisition by offering information portals.
\end{abstract}

Keywords: information exploration, information search, information retrieval, risk communication.

\section{Introduction}

With the growth and development of information technology there has been a diversification of the social risks to information-based society leading to complex social issues. Both businesses and individuals have been made victims of, for example, the leaking of personal information by computer viruses, or breaches of authors' copyrights due to unauthorized duplication. These social issues involve a complex assortment of risks regarding security, loss of privacy, erosion of user-friendliness, and management. In order to solve these issues, it is essential to consider how to reduce these various risks, and how to resolve the opposing interests of decision-makers, participants and stakeholders.

Solving these complex social issues does not merely require an analysis of the risks involved. An exchange of information and opinions-information-sharing and communication-among people either directly or indirectly related to the risks is also important so as to prevent misunderstandings or insufficient understanding among participants. A risk-communication process is therefore essential to build a consensus among participants with differing standpoints and perspectives, such as decisionmakers, stakeholders and specialists [1]. 
However, there many are cases when it is difficult for participants with sparse specialist knowledge to grasp the structure of the risks, trade-offs among various risks, and solution space. This means that risk communication cannot proceed smoothly. Summarizing these problematic points from the perspective of participants, the following three points may be stated: (1) it is not known what kind of perspectives are required for information gathering, (2) it is not known what the techniques are for revealing the kind of information necessary to understand the risks, and (3) it is not known how the obtained information stands when regarded from different perspectives.

In order to solve these problems, it is necessary to consider systems that support the determination of the combination of risk countermeasures and individual desires, and enable participants to gather essential information smoothly based on incomplete initial information. We are therefore currently developing a Multiplex Risk Communicator (MRC) as a risk-communication support tool in order to resolve the issues of social risk and social consensus building in an information-based society [1].

In this paper, we present a support system for participants to smoothly obtain the information needed to identify and evaluate their optimal combination of countermeasures using the portal system of the MRC's participant-support component. The characteristic features of the proposed system include the pattern-based breakdown of the steps in information gathering, the efficient gathering of information that participants require by means of a smooth migration between each of the information gathering methods, and the facilitation of planning in order to support the identification and evaluation of an individual's optimal combination of risk countermeasures. The utility of the system was validated experimentally.

\section{The Risk-Communication Problem}

\subsection{Characteristics of Risk Communication}

Risk communication is an interactive process whereby decision makers, participants, specialists and facilitators exchange opinions and information regarding risks, and communicate their intentions mutually. The objective in this paper is to present an interactive process for planning solutions using the MRC [1] [2][3] as a means of risk communication.

\subsection{Outline of the Multiplex Risk Communicator}

Until now, risk has been thought of as a single entity, but in fact there is a multiplicity of risks, and in order to establish risk countermeasures it is also necessary to obtain a consensus among the interested parties. The MRC is currently being developed as a tool for supporting the acquisition of an optimal combination of countermeasures while considering the various risks against this background, and planning communication between multiple participants.

The MRC supports the following four basic processes: (1) analyzing the risks according to a consideration of the tradeoffs among multiple risks, (2) selecting an optimal combination of countermeasures from multiple possible combinations, (3) identifying both the information needed for each of the participants' desired combination of countermeasures, and corresponding information gathering methods, and (4) building a consensus among the participants, decision makers, interested parties and 
specialists. The MRC specialist input/output component supports the basic processes numbered (1) and (2), and the MRC participant-support component supports those numbered (3) and (4). This paper deals with the MRC participant-support component and is focused on process (3).

\subsection{Outline of Risk Communication in the MRC Participant-Support Component}

The MRC participant-support component conducts risk communication over the following three steps [4][5].

$1^{\text {st }}$ risk communication

Information is gathered in order for each participant to clarify their opinion.

$2^{\text {nd }}$ risk communication

Mutual understanding among each of the participants is developed.

$3^{\text {rd }}$ risk communication

A consensus is established among all of the participants.

This paper deals with the portal-based information-gathering support system for the $1^{\text {st }}$ risk communication occurring in the MRC's participant-support component.

\subsection{Problems Regarding Participants with Insufficient Information}

Many participants have only sparse specialist knowledge. This makes it difficult for them to comprehend the structure of the risks in their desired solution, the tradeoffs among the risks, and what kind of position the solution occupies in the space of possible solutions. It is thus also difficult for them to compare and evaluate their desired solution with respect to an optimal solution recommended by specialists.

In such a context, participants will find it difficult to understand and interpret the issues upon which the specialists based their optimal solution. The causes may be summarized as the following three problems.

Problem 1 It is common for participants with only sparse specialist knowledge to know neither what kinds of perspective are appropriate for information gathering, nor the structure formed by the risk space.

Problem 2 It is common for participants with only sparse specialist knowledge to be unable to conduct information gathering activities fulfilling the information gathering aims and objectives.

Problem 3 Participants with only sparse specialist knowledge cannot grasp the position of the information obtained when regarded from alternative perspectives and how it relates to peripheral information.

\section{Basic Principles of Problem Solving}

\subsection{Aims}

Aim 1: To provide multiple structured perspectives constituting the basic points needed to conduct information gathering in information space. 
Aim 2: By categorizing the state transitions of the participants' information gathering process, to present the information gathering objectives and to offer information appropriate to the state so as to support comparatively specific information gathering (convergent activity) rather than abstract information gathering, and also to support comparatively abstract information gathering (divergent activity) rather than specific information gathering.

Aim 3: To provide support in order to grasp an understanding of what kind of position the information obtained occupies when regarded from alternative perspectives, and how it relates to peripheral information.

\subsection{Basic Principles}

Basic principle 1: Provide a portal for the structured information constituting the basic points of information gathering in order to fulfill Aim 1.

Basic principle 2: In order to fulfill Aim 2, participants' information gathering is categorized into three steps, and information appropriate to each information gathering state is offered. Smooth transitions between different information gathering methods are also supported.

It is common for participants with sparse specialist knowledge not to have a clear knowledge of the aims and objectives of the information gathering needed to clarify their own opinions. Information gathering for this kind of participant must involve the smoothly facilitation of three information gathering activities: making clear what kind of aims and objectives are appropriate (exploration activity), making clear what kind of structure is represented by obtained information according to a given perspective and what kind of peripheral information exists (search activity), and investigating obtained information through further detailed information (retrieval activity).

Basic principle 3: In order for fulfill Aim 3, visualizations are presented showing the position of the obtained information according to multiple differing perspectives within the structure of risk information, and showing peripheral information. The changing of perspectives is made possible.

The positional relationships within the information obtained from the multiple structured information portals, and the peripheral information relating to alternative perspectives are therefore represented visually. The promotion of convergent and divergent information gathering activities is also supported, by facilitating the changing of perspectives.

\subsection{Definition of the Information Gathering Steps (Basic principle 1)}

The following three information gathering steps are defined. The content of the information presented at each step is also shown.

1. Information exploration: objective-setting step

2. Information search: search step, for information related to the objectives

3. Information retrieval: examination step 
Information is presented as appropriate, according to each of the three information gathering steps mentioned above.

\subsection{Overview of the Information Portals (Basic principles $2 \& 3$ )}

The information portals described below which gather relevant information in a single place, are used as tools to support participants' understanding of information sources from various perspectives.

a. Portals effective for grasping risk structure

- $\quad$ Risk-analysis structure portal (Fault Tree Analysis (FTA), Event Tree Analysis (ETA), etc.)

b. Portals effective for grasping the tradeoffs among risks and the solution space

- Solution space structure portal

- $\quad$ Solution space search process portal (archive history)

c. Portals effective for studying risk

- Document structure portal (countermeasures, assumed risks, etc.)

The information portals are not used in isolation, but rather the following advantages may be achieved by combining a number of information portals and shifting between them.

1. An understanding of the risks is helped by visually establishing the relationships within the information obtained from a variety of perspectives, based on a grasp of the risk structure, the tradeoffs among multiple risks, and the solution space.

2. Convergence and divergence of information gathering activities may be freely conducted according to participants' concerns and interests, by using multiple information portals, and without being limited to a single information gathering step.

By using multiple structured information portals, participants may deepen their understanding of information obtained for the purpose of grasping the positional relationships of the obtained information within the structure of a portal, and the positional relationships of the obtained information according to different perspectives (portals). Thus, by using the information portals and conducting each information gathering step, participants may grasp an understanding of the related information they require, the risk structure, the solution space, and the tradeoffs among multiple risks.

\section{The Specific Support Procedure Occurring in the $1^{\text {st }}$ Risk Communication}

The risk communication in the MRC's participant-support component is conducted using the participants' desired solutions and compromise constraint conditions 
obtained in the $1^{\text {st }}$ risk communication. In this $1^{\text {st }}$ risk communication, desired solutions and compromise constraint conditions that reflect the participants' opinions are established by gathering information for participants to clarify their own opinions. The support process for participants' information gathering in the $1^{\text {st }}$ risk communication is proposed.[4].

According to the support process, participants make use of information portals in order to clarify their own opinions, and make state transitions through the steps involved in gathering information appropriate for the information-gathering aims and objectives.

The following steps are conducted during the exploration phase: (1) presenting what kind of information can be obtained from each portal facilitates the gathering of information involved in the objective setting steps, and (2) presenting keywords for relevant portals facilitates the gathering of information involved in the objective setting steps.

Following the exploration phase, the input for the search phase is based on the portals used for exploration. The gathering of information related to the objectives is thus conducted in the search phase by presenting structured information from the portals, and information related to the area under focus as required using overlays.

Following the search phase, the input for the retrieval phase is generated by selecting refining keys and determining the area under focus. By presenting detailed information related to the refining keys and area under focus during the retrieval phase, information is gathered for the steps of the investigation. Participants conduct information gathering activities in the three phases described above, while repeating the state transitions of each information gathering step based on their own queries. Also, while refining the information required, participants evaluate their desired solutions and validate their applicability. As a result, the desired solution and compromise constraint conditions constituting each participant's own opinions are established.

\section{Empirical Validation}

\subsection{Experimental Objectives}

The experimental objectives were to validate whether or not participants could establish the following points (in their own opinions) by conducting the $1^{\text {st }}$ risk communication in the MRC's participant-support component portal system.

(1) Determining the objective function and items in the constraint conditions [2] [3]

Objective function:

Minimum total cost of the "sum of the cost of the countermeasures and the risk of personal information leaks"

Constraint conditions:

(a) cost of countermeasures (Japanese Yen)

(b) privacy burden for employees (\%)

(c) user-friendliness burden for employees (\%)

(d) personal information leak rate $(\%=$ cases per year $)$ 
(2) Election of the set of countermeasures [2][3]

8 countermeasures were elected for the set

1. Could the desired solution be established?

2. Could the compromise constraint conditions be established?

\subsection{Preconditions}

The issue of personal information leaks was treated as an object of the risk communication. The subjects were 10 male university students in their early twenties, and were assigned roles as managers or employees.

\subsubsection{Establishing the Assumed Target Enterprise}

The target business was assumed to be a service provider company with $1,000,000$ personal information retained.

\subsubsection{Preconditions of the Risk-Analysis Background}

The risk analysis procedure regarding personal information leaks was conducted as follows .

\subsubsection{Establishing the information portals}

The information portals comprise a set of four portals: the countermeasures portal, the assumed-risk portal, the scatter-plot portal, and the FTA portal.

\subsection{Experimental Results}

Managers' query: Given the security revolution, what cost of countermeasures should be accommodated in response to risks? Managers followed an information gathering process in order to resolve their query. It reveals that after going through the information gathering process, managers ultimately selected the solution endorsed by specialists as their desired solution. Also, they selected a leak rate of at most $20 \%$, and a cost of at most 80 million yen for countermeasures as constraint conditions.

An example information search shows that by using the scatter-plot portal, participants were able to investigate what kind of structure the whole solution space takes when defined according to two constraint-condition axes, where the optimal and desired solutions exist in solution space, and what kind of solutions exist in the surrounding area of solution space.

Example overlay of information portals shows that by using a superposition of multiple information portals, participants were able to grasp an understanding of what other sets of countermeasures exist, what kind of detailed information is available regarding other sets of countermeasures, and where in solution space the sets of countermeasures incorporating the desired countermeasures exist. 


\section{Discussion of Experimental Results}

Among the various results of the information gathering process, managers mainly centered their information gathering activities on the scatter-plot portal. They developed an understanding of the risk structure through gathering a quantitative perspective on the solution space by searching it using the scatter-plot portal. In addition, by way of auxiliary information gathering, they used the FTA portal to search for information from a qualitative perspective regarding the causes of risks and positional relationships among the countermeasures. They also used the countermeasures portal and assumed-risk portal to retrieve information about the risks and countermeasures, making transitions among each of the portals to solve problems 1,2 and 3.

It was possible to conduct smooth transitions during information gathering according to the process described above using basic principles 1,2 and 3. Managers were able to resolve their initial queries, and establish their desired solution and compromise constraint conditions.

\section{Conclusion}

In this paper we proposed a support process for smooth information gathering aimed at allowing participants with sparse specialist knowledge to grasp the risk structure, the tradeoffs among multiple risks, and the solution space itself in the context of the multiplex risk communicator.

A characteristic of the proposed method is the pattern-based categorization of participants' information gathering process. In addition, the smooth transitions among each of the information gathering steps were planned in order to make the gathering of information that participants regard as essential, smoother and more efficient.

The utility of the proposed method was validated empirically by conducting experiments using the issue of personal information leaks as the object of an example risk communication.

\section{Acknowledgements}

We received much advice regarding the participant-support component of the multiplex risk communicator (MRC) from members of the multiplex risk communicator working group, to whom we are very grateful, and we are also very grateful to the people who took part in our experiments.

This research is supported in the activity of security and safety working group in applied security forum, and is studied in the research on "Challenges in identifying vulnerabilities hidden in our highly sophisticated information society and exploring solutions" of the Research Institute of Science and Technology for Society in Japan Science and Technology Agent. We thank Dr. Murayama and Dr. Yoshiura for their useful comments and advices 


\section{References}

1. Sasaki, R., Ishii, S., Hidaka, Y., Yajima, H., Yoshiura, H., Murayama, Y.: Development Concept for and Trial Application of a Multiple Risk Communicator. In: 5th IFIP Conference e-Commerce, e-Business, and e-Government (I3E (2005)

2. Sasaki, R.: Sturdy of Relationship between security and ptrotekution of personal information SITE2003-14, pp. 1-6 (2003)

3. Sasaki, R.: Compatibility and Conflict between security and protekution of personal information. Cyber Security Management 6, 20-24 (2003)

4. Yajima, H., et al.: Support System for Decision Maker in Multiple Risk Communication. CHI, vol. 7 (2005)

5. Yajima, H., Matsumoto, S., Sasaki, R.: Proposal of Risk Communication Supporting Method in Multiplex Risk Communicator. In: Proceeding of 11th IEEE International Conference on Emerging Technologies and Factory Automation, pp. 255-260 (2006) 